\title{
Amplification, proto-oncogènes et cancers humains
}

L'amplification est un moyen d'activation de certains proto-oncogènes dans des cancers humains. Qu'elle apparaisse sous forme intra- ou extrachromosomique, la région génomique amplifiée, ou amplicon, semble résulter d'événements initiaux de recombinaison qui pourraient être communs à d'autres types d'altérations génétiques (délétions, translocations). Souvent de grande taille, l'amplicon peut contenir plusieurs gènes adjacents à celui dont l'amplification - et la sur-expression confèrent un avantage sélectif aux cellules qui le portent. Le rôle éventuel de ces gènes co-amplifiés dans la progression tumorale reste à déterminer. Si l'amplification de $\boldsymbol{N}$-myc dans les neuroblastomes semble avoir une réelle valeur pronostique, la situation est plus controversée pour d'autres couples proto-oncogène/tumeur.

\section{Pierre Szepetowski Danielle Perucca-Lostanlen Patrick Gaudray}

\section{ADRESSE}

P. Szepetowski : interne de recherche médicale. D. Perucca-Lostanlen : chargée de recherche à l'Inserm. P. Gaudray : directeur de recherche au Cnrs. Laboratoire de génétique moléculaire des cancers humains, UFR de médecine, avenue de Valombrose, 06034 Nice Cedex,

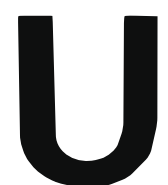

n des moyens utilisés par certaines cellules pour répondre à une demande accrue en un produit donné est d'augmenter le nombre de copies du gène codant pour ce produit. Ce phénomène, parfois appelé de manière restrictive " amplification génique ", est mieux défini par le terme " amplification d'ADN ", dans la mesure où la taille de la région chromosomique amplifiée est, dans la grande majorité des exemples connus, bien plus importante que la seule unité transcriptionnelle dont l'amplification est requise.

\section{L'amplification d'ADN est un phénomène largement répandu}

L'amplification d'ADN est une propriété largement répandue dans le monde biologique; elle intervient dans le développement normal de plusieurs espèces : l'ADN ribosomal est fortement amplifié au cours de l'oogenèse chez Xenopus laevis ; chez Drosophila melanogaster, les deux groupes de gènes codant pour les protéines chorioniques sont amplifiés dans les cellules ovariennes afin de permettre la fabrication de l'enveloppe de l'œuf.

L'amplification d'ADN dans une cellule peut être un moyen d'adaptation à de nouvelles conditions environnementales : on détecte ainsi des duplications de gènes dans des bactéries poussant en milieu sélectif. De même, l'amplification de régions d'ADN contenant un gène permettant de résister à un agent de sélection est-elle observée in vitro dans divers systèmes eucaryotes : résistance au méthotrexate (MTX) et amplification du gène codant pour la dihydrofolate réductase (DHFR), résistance au $\mathrm{N}$-(phosphonacétyl)-Laspartate (PALA) et amplification du gène codant pour la protéine trifonctionnelle CAD (carbamylphosphate synthétase/aspartate transcarbamylase/dihydroorotase), en sont deux exemples [1]. 
L'amplification du gène $D H F R$ est aussi un mécanisme de résistance au MTX observé cliniquement [2]. Dans un tout autre domaine, la résistance de certains moustiques aux insecticides organophosphorés est due à un fort niveau d'amplification d'un gène codant pour une enzyme de détoxification, l'estérase B1 [3]. Un gène codant pour une cholinestérase est amplifié chez deux hommes de la même famille exposée de longue date aux organophosphorés [4].

L'amplification d'ADN peut parfois être visualisée en cytogénétique, sous deux formes différentes. La première, extrachromosomique, correspond aux chromosomes minutes. Structures circulaires super-enroulées, souvent appelées doubles-minutes (Dm) du fait de leur aspect dupliqué, ces élé- ments se répliquent de manière autonome et, ne possédant pas de centromère, sont distribués inégalement lors de la mitose : en absence de pression sélective, ils sont rapidement perdus dans la population cellulaire. L'autre forme visible est constituée par les HSR (homogeneously staining region) ou ECR (expanded chromosome region), régions intrachromosomiques étendues d'ADN amplifié, dont la ségrégation se fait normalement à chaque mitose, et qui sont donc conservées de manière relativement stable, même si la sélection n'est plus appliquée. Amplification intrachromosomique n'est pas synonyme d'amplification in situ: une ECR localisée sur un chromosome peut correspondre à une amplification de séquences provenant d'un autre chromosome [1].

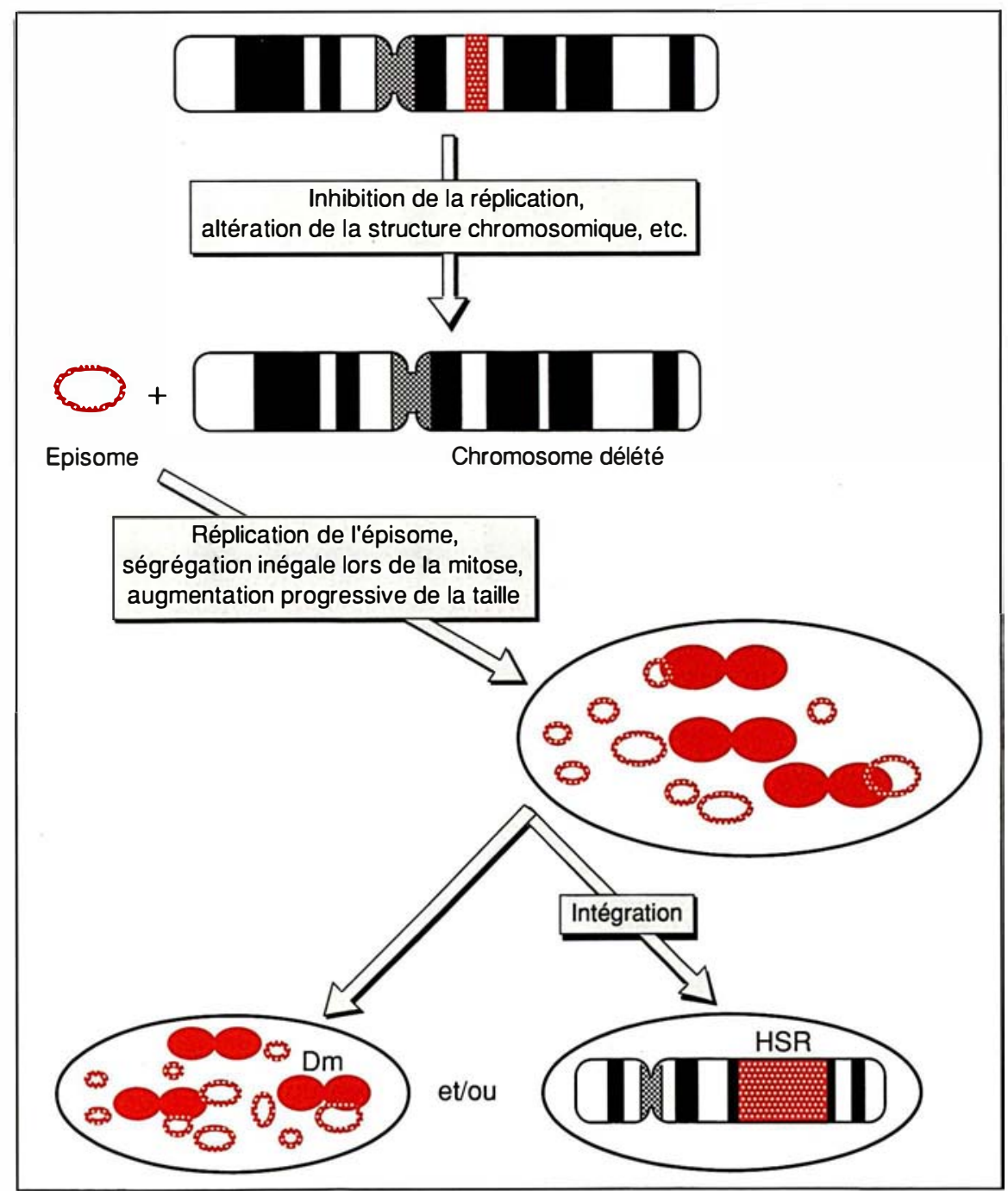

Figure 1. Schéma possible des étapes conduisant à la formation des chromosomes minutes (Dm) et des régions chromosomiques étendues (HSR).
Ces deux types de marqueurs d'amplification sont retrouvés dans différentes cellules et lignées cellulaires, issues de tumeurs malignes. Ils représentent deux formes possibles d'une même séquence d'ADN amplifié, ces deux formes ne semblant pas coexister dans une cellule pour une même séquence d'ADN, à un moment donné. D'une manière générale, il semble que les Dm soient les formes cytogénétiques prépondérantes d'amplification dans les tumeurs humaines, in vivo [5].

\section{L'amplification d'ADN est un phénomène complexe}

Les séquences d'ADN amplifiées sont très variables : leur taille, le type de réarrangements chromosomiques et les modifications fréquentes dont elles sont les cibles diffèrent selon les cellules, les conditions et les systèmes expérimentaux utilisés. La longueur de l'unité amplifiée, ou amplicon, qui est répétée un certain nombre de fois dans la région amplifiée, peut aller de quelques dizaines à plusieurs milliers de kilobases, dépassant généralement la taille du gène - ou des gènes - dont l'amplification est sélectionnée. De nombreuses études ont montré que l'ADN des régions amplifiées peut avoir une structure bien plus complexe que celle d'une simple répétition linéaire d'unités identiques. Plusieurs hypothèses ont été, et sont encore avancées pour tenter d'expliquer les mécanismes de l'amplification [6]. Les deux plus classiques font appel à un échange inégal de chromatides-sœurs, comme cela semble être le cas chez Escherichia coli, ou à une sur-réplication au cours d'un cycle cellulaire, comme c'est le cas pour les gènes chorioniques chez la drosophile.

La taille des chromosomes minutes peut augmenter progressivement, ce qui laisse penser qu'ils pourraient provenir de précurseurs non visibles en cytogénétique (figure 1). Un des événements initiateurs de l'amplification pourrait être la formation de séquences d'ADN extrachromosomiques, structures nommées épisomes [5]. Ces épisomes seraient créés par un événement de recombinaison intrachromosomique au sein d'une 


\section{RÉFÉRENCES}

1. Schimke RT. Gene amplification in cultured cells. J Biol Chem 1989; 263 : 5989-92.

2. Curt GA, Carney DN, Cowan KH, et al. Unstable methotrexate resistance in human small-cell carcinoma associated with double minute chromosomes. $N$ Eng J Med 1983 ; 308 : 199-202.

3. Mouchès C, Pasteur N, Bergé JB, et al. Amplification of an esterase gene is responsible for insecticide resistance in a California Culex mosquito. Science 1986; 233 : 778-80.

4. Prody CA, Dreyfus $\mathrm{P}$, Zamir R, Zakut H, Soreq H. De novo amplification within a " silent " human cholinesterase gene in a family subjected to prolonged exposure to organophosphorus insecticides. Proc Natl Acad Sci USA 1989 ; 86 : 690-4.

5. Wahl GM. The importance of circular DNA in mammalian gene amplification. Cancer Res 1989 ; 49 : 1333-40.

6. Stark GR, Debatisse M, Giulotto E, Wahl GM. Recent progress in understanding mechanisms of mammalian DNA amplification. Cell 1989 ; 57 : 901-8.

7. Carroll SM, DeRose ML, Gaudray P, et al. Double minute chromosomes can be produced from precursors derived from a chromosomal deletion. Mol Cell Biol 1988 ; $8: 1525-33$.

8. Hunt JD, Valentine M, Tereba A. Excision of $N$-myc from chromosome 2 in human neuroblastoma cells containing amplified $N$ myc sequences. Mol Cell Biol 1990; 10 : 823-29.

9. Saito I, Groves R, Giulotto E, Rolfe M, Stark GR. Evolution and stability of chromosomal DNA coamplified with the CAD gene. Mol Cell Biol 1989 ; 9 : 2445-52.

10. Brodeur GM, Fong C. Molecular biology and genetics of human neuroblastoma. Cancer Genet Cytogenet 1989 ; 41 : 153-74.

11. Seeger RC, Brodeur GM, Sather H, et al. Association of multiple copies of the $N$ myc oncogene with rapid progression of neuroblastomas. $N$ Eng J Med $1985 ; 313$ : 1111-6.

12. Delidakis C, Kafatos FC. Amplification enhancers and replication origins in the autosomal chorion gene cluster of Drosophila. $E M B O$ J $1989 ; 3$ : 891-901.

13. Fong CT, Dracopoli NC, White PS, $e$ al. Loss of heterozygosity for chromosome $1 \mathrm{p}$ in human neuroblastomas : correlation with $N-m y c$ amplification. Proc Natl Acad Sci USA $1989 ; 86: 3753-7$.

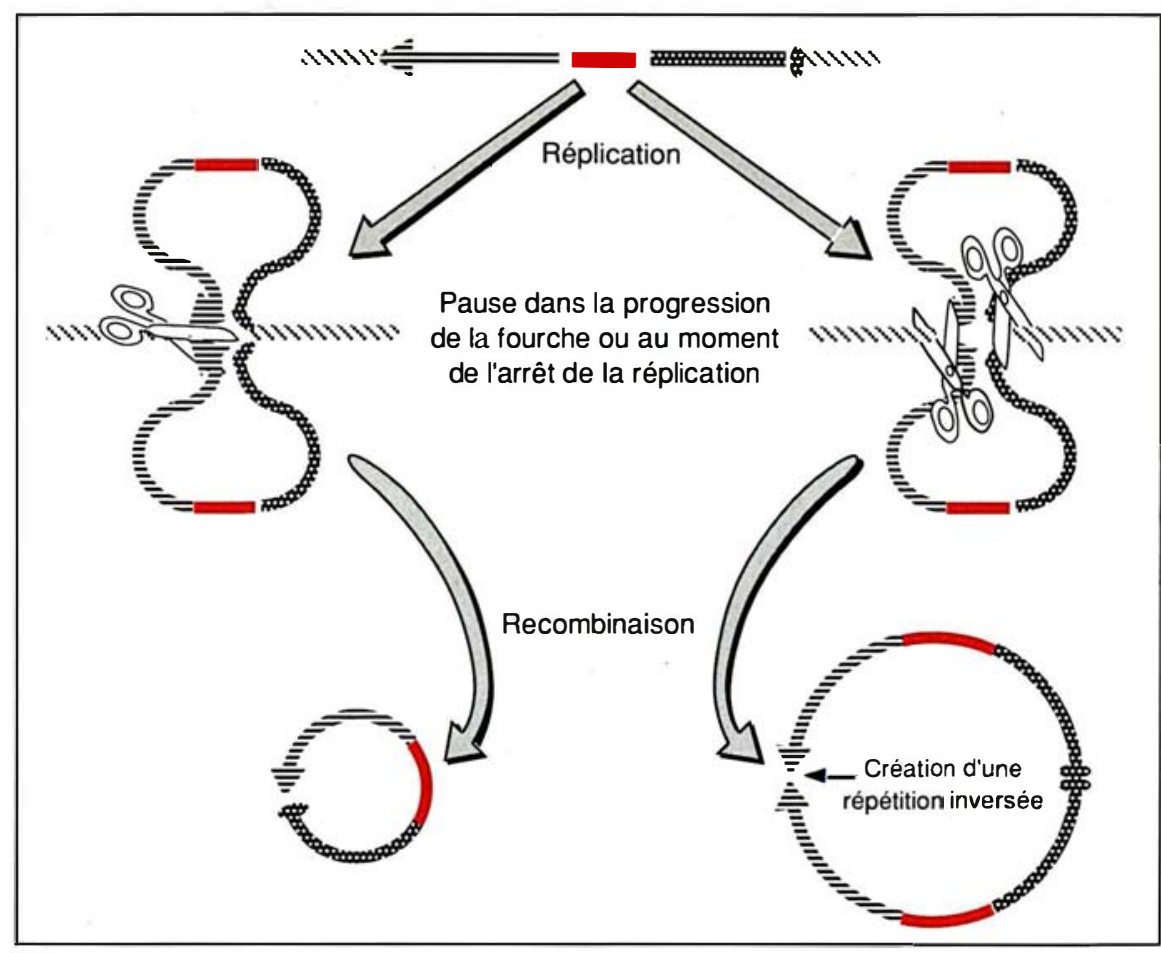

Figure 2. Formation des épisomes au sein d'une boucle de réplication, par recombinaison intrachromosomique (d'après Wahl, 1989).

boucle de réplication (figure 2), créant une délétion dans le chromosome et une molécule circulaire extrachromosomique ; celle-ci, si elle contient une origine de réplication (au moins) et un gène (au moins) donnant à la cellule un avantage sélectif, va conduire, du fait de la ségrégation inégale lors de la mitose, à l'amplification du ou des gènes en question. Des épisomes ont effectivement été identifiés dans plusieurs systèmes cellulaires, et des études récentes [7, 8] amènent à faire un lien entre délétion et amplification.

Les différents mécanismes évoqués pourraient tous être impliqués, mais dans des systèmes différents; la variété des structures et des localisations des unités amplifiées va dans ce sens. Cette variété pourrait aussi signifier que la résolution d'un événement initial unique s'opérerait de différentes manières. Un niveau élevé d'amplification est atteint en plusieurs étapes au cours desquelles de nombreux réarrangements sont possibles [9]. Ainsi l'amplification d'ADN est-elle un phénomène dynamique. On l'observe à un moment précis de l'évolution d'une population cellulaire, mais l'importance réelle d'un état d'amplification particulier, dans l'histoire de ces cellules, ne se situe pas forcément dans l'instant présent.

\section{Activation des proto-oncogènes par amplification : exemple de $\mathrm{N}$-myc}

Les séquences d'ADN amplifiées dans diverses tumeurs malignes contribuent probablement au phénotype tumoral, le ou les gènes qu'elles contiennent étant susceptibles, dans certaines conditions, de conférer un avantage sélectif aux cellules tumorales. Deux grandes catégories de gènes sont trouvées amplifiées dans les cellules cancéreuses : les gènes de résistance aux drogues chimiothérapiques, d'une part (presque toujours in vitro) ; les proto-oncogènes, d'autre part.

De nombreux proto-oncogènes ont été associés à divers cancers humains. Leur activation en oncogènes est due soit à des altérations structurales de leur séquence codante (insertion, réarrangement, délétion, mutation ponctuelle), soit à des erreurs quan- 


\section{TABLEAU I}

\section{Liste (non exhaustive) des proto-oncogènes amplifiés dans diverses affections néoplasiques}

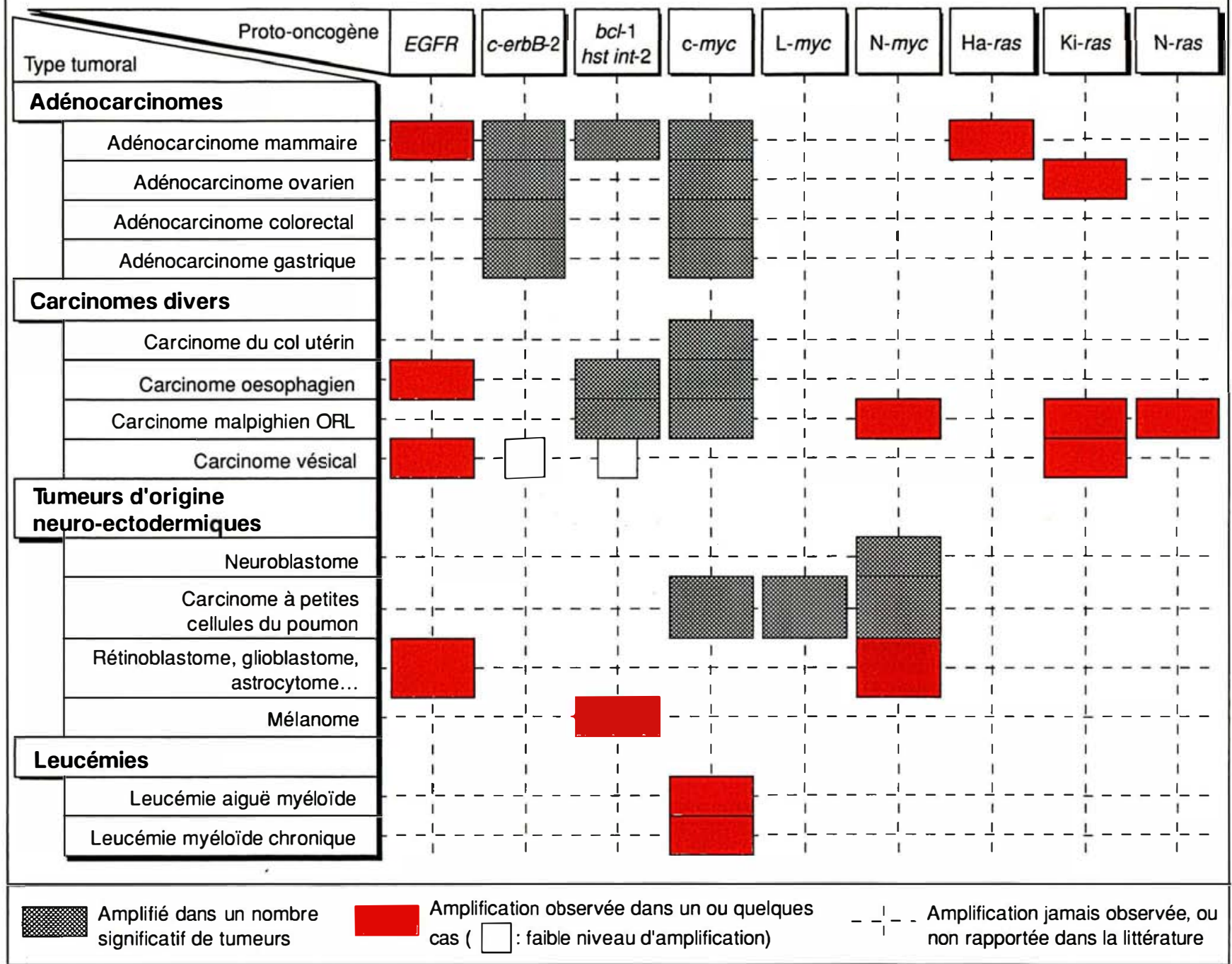

titatives ou qualitatives de leur expression. L'augmentation de l'expression par amplification, qui conduirait à l'émergence d'une souspopulation de cellules tumorales possédant des potentialités malignes accrues, représente donc un moyen d'activation de certains protooncogènes.

Un exemple maintenant classique est celui de l'amplification du protooncogène $N$-myc. $N$-myc a été primitivement identifié dans les neuroblastomes humains, où il est souvent amplifié dans le tissu tumoral comme dans des lignées cellulaires dérivées de ce type de tumeur. Il existe une $m / s n^{\circ} 1$, vol. 7 , janvier 91 forte corrélation entre l'amplification de $N$-myc, l'hyperexpression qui en résulte et le stade tumoral observé [10] : l'amplification de $N$ - $m y c$ est rarement observée dans les stades I et II de la maladie, alors qu'elle est fréquente $-30 \%$ des cas environ - dans les stades III et IV. L'amplification de $N-m y c$ a été associée à un mauvais pronostic vital [11], tant qualitativement (pour un même stade tumoral, la présence de l'amplification est de plus mauvais pronostic que son absence) que quantitativement (plus haut est le niveau d'amplification, plus courte est la durée de vie). Le niveau d'amplifi- cation de $N$-myc pourrait ainsi être un bon marqueur de pronostic dans les neuroblastomes humains, permettant d'affiner les choix thérapeutiques à faire, notamment dans les stades tumoraux apparemment favorables.

Si l'amplification de $N$-myc n'est pas spécifique des neuroblastomes (Tableau I), elle semble limitée à un petit nombre de types tumoraux particuliers. De même, l'amplification de $L-m y c$ n'a été décrite que pour les carcinomes anaplasiques à petites cellules du poumon. En revanche, celle de $c-m y c$ est retrouvée dans une grande variété de cellules tumorales, que ce soit in vitro ou in vivo, sans 


\section{RÉFÉRENCES}

14. Wright JA, Smith HS, Watt FM, Hancock MC, Hudson DL, Stark GR. DNA amplification is rare in normal human cells Proc Nall Acad Sci USA 1990 ; 87 : 1791-5.

15. Yokota J, Yamamoto T, Miyajima N, et al. Genetic alterations of the c-erbB-2 oncogene occur frequently in tubular adenocarcinoma of the stomach and are of ten accompanied by amplification of the $v$-e $t b-A$ homologue. Oncogene 1988 ; 2 : 283-7.

16. Perucca D, Szepetowski P, Simon MP Gaudray P. Molecular genetics of human bladder carcinomas. Cancer Genet Cytogenet 1990 ; 49 : 143-6.

17. Adnane J, Gaudray $P$, Simon MP, Simony-Lafontaine J, Jeanteur P, Theillet C. Proto-oncogene amplification and human breast tumor phenotype. Oncogene $1989 ; 4: 1389-95$

18. Berger MS, Locher GW, Saurer S, et al. Correlation of $c-e r b B-2$ gene amplification and protein expression in human breast carcinoma with nodal status and nuclear grading. Cancer Res $1988 ; 48: 1238-43$.

19. Slamon DJ, Clark GM, Wong SG Levin WJ, Ullrich A, McGuire WL. Human breast cancer : correlation of relapse and survival with amplification of the HER-2/neu oncogene. Science 1987 ; 235 : 177-82.

20. Guérin $M$, Barrois $M$, Terrier $M J$, Spielmann M, Riou G. Overexpression of either $c$-myc or $c$-erbB-2/neu proto-oncogenes in human breast carcinomas : correlation with poor prognosis. Oncogene Res 1988 ; 3 : 21-31.

21. Ali LU, Campbell G, Lidereau R, Callahan $R$. Lack of evidence for the prognostic significance of c-erbB-2 amplification in human breast carcinoma. Oncogene Res $1988 ; 3: 139-46$.

22. Zhou DJ, Ahuja H, Cline MJ. Protooncogene abnormalities in human breast cancer : c-erbB-2 amplification does not correlate with recurrence of disease. Oncogene $1989 ; 4$ : 105-8.

23. Callahan R. Genetic alterations in primary breast cancer. Breast Cancer Res Treat $1989 ; 13: 191-203$.

24. Rice GC, Hoy C, Schimke RT. Transient hypoxia enhances the frequency of dihydrofolate reductase gene amplification in Chinese hamster ovary cells. Proc Natl Acad Sci USA 1986 ; 83 : 5978-82. pourtant que l'on retrouve une corrélation entre le nombre de copies du gène et le stade tumoral ou le pronostic de la maladie. Dans les carcinomes anaplasiques à petites cellules du poumon, l'un quelconque des trois gènes miyc précités peut être amplifié ; ce n'est pas le cas dans les neuroblastomes, où ni $c-m y c$ ni $L-m y c$ ne sont amplifiés. Il est possible que l'évolution d'un certain nombre de neuroblastomes fasse appel à une fonction spécifique de $N-m y c$, que ne posséderaient ni $L-m y c$ ni $c-m y c$. De plus, si leurs séquences nucléotidiques sont homologues, les gènes myc possèdent certainement des environnements génétiques très différents. Les séquences voisines d'un gène peuvent en partie déterminer la fréquence à laquelle il est amplifié : cela semble être le cas lors de l'amplification des gènes chorioniques de la drosophile, où plusieurs éléments " activateurs" en cis de l'amplification ont été identifiés récemment [12]. Reste cependant à montrer que ce type d'activation est impliqué dans un système moins " programmé " que ne l'est l'embryogenèse de la drosophile.

Des études récentes font apparaître une corrélation étroite entre l'amplification de $N$-myc, situé sur le bras court du chromosome 2 , et la perte d'information génétique sur la partie distale du bras court du chromosome 1 , autre lésion fréquente dans les neuroblastomes [13]. L'interprétation précise de cette observation nécessitera sa confirmation sur un grand nombre de tumeurs et son étude détaillée. Néanmoins, il est tentant de supposer que ces deux événements puissent être les conséquences d'un mécanisme commun, ou que l'un puisse être cause de l'autre. Amplification et délétion pourraient représenter deux manifestations d'une plus grande instabilité du génome de certaines souspopulations de cellules cancéreuses. Les cellules cancéreuses pourraient avoir acquis la capacité d'amplifier l'ADN, contrairement aux cellules normales [14], grâce à l'expression de gènes restant à déterminer. Est-ce l'amplification d'un proto-oncogène particulier qui est un élément défavorable ? ou est-ce la présence d'une amplification per se qui représente un facteur de mauvais pronostic ? La plus grande instabilité du génome de ces cellules permet-elle une adaptabilité plus rapide aux conditions extérieures et une meilleure réponse aux pressions de sélection diverses s'appliquant sur la tumeur ou sur une partie de celle-ci?

\section{La signification clinique de I'amplification est loin d'être simple}

Plusieurs autres proto-oncogènes sont amplifiés dans divers types de cancers, avec une spécificité tumorale plus ou moins grande (Tableau I) ; inversement, certains types de tumeurs présentent plusieurs protooncogènes amplifiés, soit isolément (chez des malades différents), soit ensemble (chez le même malade), dans le même tissu tumoral, voire dans les mêmes cellules. Il semble très probable que le phénomène d'amplification dépende, entre autres causes, du proto-oncogène en question (et des régions chromosomiques adjacentes), de l'organe atteint (et parfois plus précisément du type histologique tumoral, telle l'amplification de c-erbB-2 dans les adénocarcinomes tubulaires gastriques [15]) et des interactions entre les deux. L'amplification de $c-m y c$ est ainsi retrouvée dans des types de cancers apparemment sans relations entre eux ; de même, celle de c-erbB-2 est assez fréquemment répandue, quoique principalement limitée aux adénocarcinomes. Cependant, la plupart des proto-oncogènes connus à ce jour semblent rarement amplifiés dans les cancers humains, voire pas du tout. Inversement, si certains types de cancers humains semblent constituer un terrain peu propice au phénomène d'amplification, comme par exemple les carcinomes vésicaux [16] - même si l'on ne peut exclure que des gènes encore inconnus $y$ soient fréquemment amplifiés - , il existe d'autres tissus cancéreux au sein desquels l'amplification de certains gènes est, sinon favorisée, du moins possible et effective. De ce point de vue, les cancers du sein sont un bon exemple de tissu tumoral dans lequel plusieurs proto-oncogènes peuvent être amplifiés. 
Représentant les cancers féminins les plus fréquents dans les pays occidentaux, les tumeurs mammaires malignes ont été intensivement étudiées, d'un point de vue tant fondamental que clinique. Environ $50 \%$ des cancers primitifs du sein possèdent au moins un proto-oncogène amplifié. Si l'amplification de certains protooncogènes peut paraître anecdotique compte tenu de la faible fréquence de l'événement, en revanche trois loci du génome humain sont fréquemment amplifiés dans les cancers primitifs du sein : $c-m y c, c-e r b B-2$ et, sur le bras long du chromosome 11, la région $11 \mathrm{q} 13$ comprenant les gènes hst et int-2 (Tableau I) [17]. Tant la fréquence de ces amplifications que leur signification varient suivant les études publiées. Ainsi l'amplification de $c$-erbB-2 peut être retrouvée dans 10 à $30 \%$ des cas. Cette amplification a pu être statistiquement corrélée à l'envahissement ganglionnaire [18], à un mauvais pronostic [19] ou à l'absence de récepteurs hormonaux [20], mais certaines études ne montrent aucune corrélation avec les facteurs pronostiques connus, ni avec l'évolution de la maladie [21, 22]. Le même type de controverse existant pour les amplifications de $c-m y c$ et du locus int-2/hst, il est difficile de savoir quelle est la réelle signification de chacune de - ou de toutes - ces amplifications [23]. Plusieurs facteurs peuvent permettre d'expliquer les différences constatées : problèmes techniques liés aux multiples altérations génétiques présentes dans les tumeurs du sein ; hétérogénéité des populations étudiées et des prélèvements chirurgicaux disponibles [17] ; différences dans les stratégies thérapeutiques utilisées, pouvant influer sur le taux d'apparition de métastases ou de récidives loco-régionales.

Un autre facteur d'erreurs, commun à toutes les études, mais dépendant aussi de la qualité du prélèvement, est le fait que l'amplification observée au niveau de l'ADN d'un prélèvement tissulaire de tumeur maligne n'est jamais qu'une moyenne des niveaux d'amplification réels atteints dans chaque cellule, cancéreuse ou non, présente dans le prélèvement étudié. Le niveau d'amplification observé sur la totalité de l'ADN présent est donc quasiment toujours

$\mathrm{m} / \mathrm{s} n^{\circ} 1$, vol. 7 , janvier 91 sous-évalué. Il y a, d'une part, contamination certaine par le stroma péri-tumoral, dans lequel les cellules ont a priori deux copies du gène étudié, et, d'autre part, contamination probable des cellules cancéreuses présentant l'amplification du gène par celles ne la présentant pas, cela d'autant plus que la tumeur sera hétérogène (figure 3). Si $\mathrm{Pa}$ est le taux de cellules du prélèvement présentant une amplification moyenne $\mathrm{Na}$, et $\mathrm{Pb}$ le taux de cellules, cancéreuses ou non, ne présentant pas cette amplification, le taux d'amplification global du prélèvement No, observé grâce aux southern blots, sera égal à No =
$\mathrm{Na} \cdot \mathrm{Pa}+1 \times \mathrm{Pb}$, soit $\mathrm{No}=(\mathrm{Na}-$ 1). $\mathrm{Pa}+1$. Un exemple de faux négatif pourrait être le suivant : $10 \%$ des cellules amplifiant 5 fois donneront un No à 1,4 , et la tumeur sera considérée sans ambiguïté comme non amplifiée. Inversement, observer un niveau d'amplification de 2 de façon reproductible, avec plusieurs contrôles, peut être considéré comme valide, puisque l'amplification réelle des cellules tumorales est probablement plus forte (notons ici qu'un taux d'amplification de 2 signifie qu'il existe quatre copies du gène dans la cellule : l'allèle " normal " et l'allèle " amplifié " trois fois).

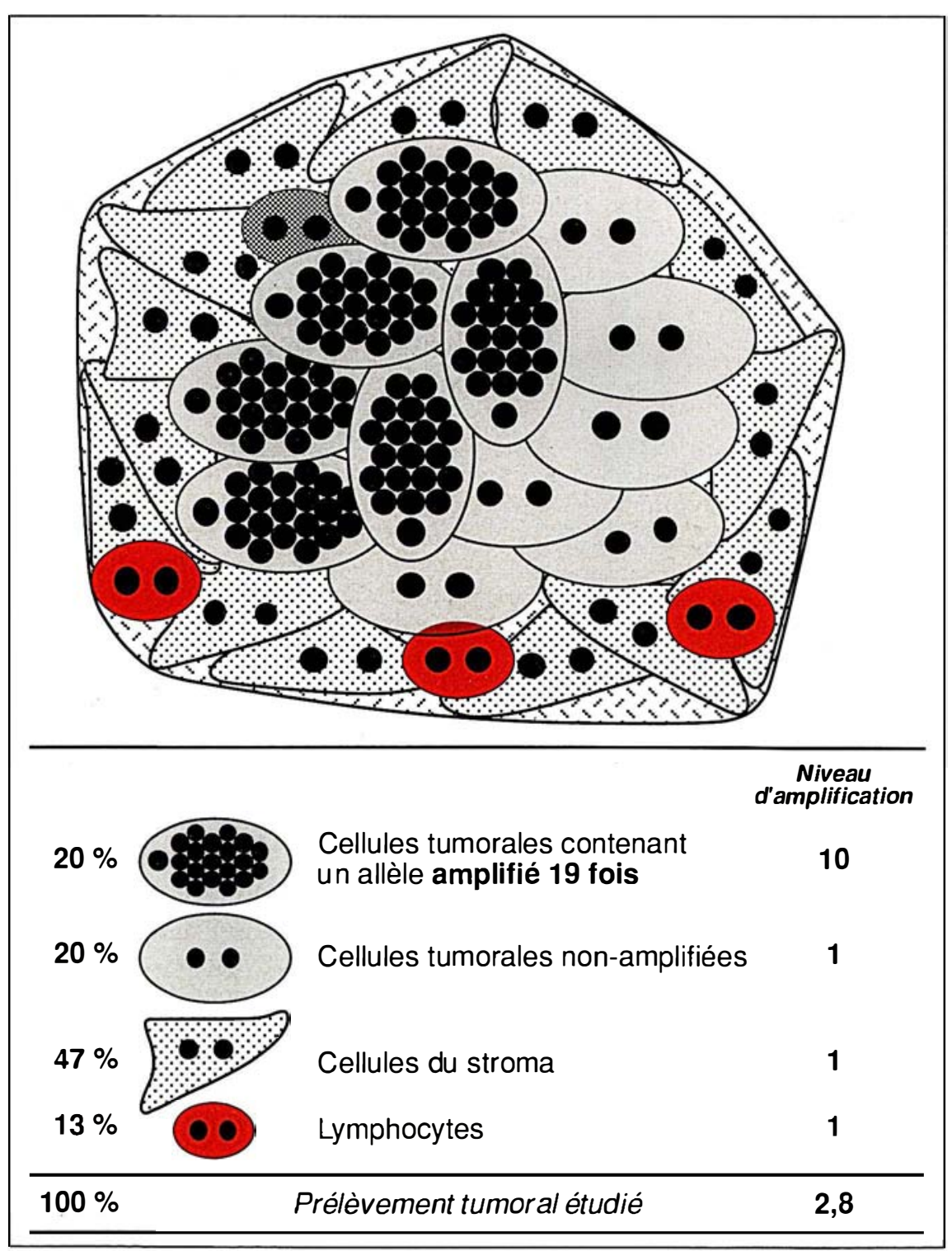

Figure 3. Exemple illustrant la sous-estimation de I'amplification dans un prélèvement tumoral. 


\section{RÉFÉRENCES}

25. Amler LC, Schwab M. Amplified $N$ $m y c$ in human neuroblastoma cells is of ten arranged as clustered tandem repeats of differently recombined DNA. Mol Cell Biol $1989 ; 9: 4903-13$

26. Slamon DJ, Godolphin W, Jones LA, et al. Studies of the HER-2/neu protooncogene in human breast and ovarian cancer. Science 1989 ; 244 : 707-12.

27. Van De Vijuer $M$, Van De Bersselaar R, Devilee P, Cornelisse C, Peterse J, Nusse R. Amplification of the neu (c-erbB-2) oncogene in human mammary tumors is relatively frequent and is often accompanied by amplification of the linked c-erbA oncogene. Mol Cell Biol 1987 ; 7 : 2019-23

28. Shtivelman E, Bishop JM. The PVT gene frequently amplifies with $\mathrm{MYC}$ in tumor cells. Mol Cell Biol 1989; 9 : 1148-54.

29. Foreman PK, Hamlin JL. Identification and characterization of a gene that is coamplified with dihydrofolate reductase in a methotrexate-resistant $\mathrm{CHO}$ cell line. Mol Cell Biol 1989 ; 9 : 1137-47.

30. Debatisse M, Robert de Saint Vincent B, Buttin G. Expression of several amplified genes in an adenylate-deaminase overproducing variant of chinese hamster fibroblasts. $E M B O J 1984 ; 3$ : 3123-7.

31. Theillet C, Adnane J, Szepetowski P, et al. Bcl-1 participates in the $11 \mathrm{q} 13$ amplification found in breast cancer. Oncogene $1990 ; 5: 147-9$.

32. Peters G, Brookes S, Smith R, Placzek $\mathrm{M}$, Dickson C. The mouse homolog of the $h s t / k-F G F$ gene is adjacent to int-2 and activated by proviral insertion in some virally induced mammary tumors. Proc Natl Acad Sci USA 1989 ; 86 : 5678-82.

33. Liscia DS, Merlo GR, Garrett C, French D, Mariani-Costantini R, Callahan R. Expression of int-2 mRNA in human tumors amplified at the int-2 locus. Oncogene $1989 ; 4: 1219-24$

34. Theillet C, Le Roy X, De Lapeyric̀re $\mathrm{O}$, et al. Amplification of FGF-related genes in human tumors : possible involvement of hst in breast carcinomas. Oncogene $1989 ; 4: 915-22$

35. Saint-Ruf C, Gerbault-Seureau $M$, Viegas-Péquignot E, Zafrani B, Cassingena $\mathrm{R}$, Dutrillaux B. Proto-oncogene amplification and homogeneously staining regions in human breast carcinomas. Genes, chromoso-
En fait, la question est de savoir si la valeur pronostique réelle de l'amplification est liée au niveau global d'amplification observé, ce qui, nous l'avons vu pour $N$ - $m y c$ dans les neuroblastomes, est parfois le cas et correspond peut-être aux tumeurs assez, voire très homogènes, ou si elle est mieux représentée par le taux réel d'amplification dans les cellules individualisées, même si elles sont peu nombreuses. Il est possible que la réponse soit différente selon le gène amplifié : si l'on conçoit qu'un facteur intracellulaire ou un récepteur membranaire doive être activé dans la cellule dont l'évolution tumorale va être favorisée, en revanche un facteur de croissance peut être sécrété par un petit nombre de cellules dans lesquelles son gène est amplifié et influencer la croissance de nombreuses autres cellules. L'étude des tissus tumoraux en hybridation in situ permettrait-elle de montrer dans quelles cellules une amplification peut être observée et de quantifier celleci ? On peut supposer l'existence de zones préférentielles d'amplification, différentes selon le gène étudié, visualisées sous forme de "points chauds " d'amplification. L'amplification d'un gène donné peut n'être nécessaire que dans un territoire tumoral bien précis et, de plus, certaines zones tumorales peuvent être sous l'influence de facteurs extrinsèques locaux favorisant les phénomènes d'amplification : ainsi l'hypoxie favorise-t-elle l'amplification du gène DHFR in vitro [24], et peut-être en est-il de même in vivo et pour d'autres couples que $D H F R /$ hypoxie.

\section{Plusieurs gènes}

\section{sur un même amplicon}

Du fait de leur grande taille, les régions amplifiées sont susceptibles de contenir plusieurs gènes adjacents à celui, ou à ceux, dont l'amplification est sélectionnée. On peut schématiquement distinguer trois types de gènes dans un amplicon:

- les gènes conférant effectivement un avantage sélectif à la cellule, dont on peut attendre qu'ils soient surexprimés, ou qu'ils l'aient été ; - les gènes "indifférents ", dont l'amplification est coïncidente avec celle du gène important, et moins fréquente que pour celui-ci dans la population des sujets atteints. Ces gènes peuvent ou non être exprimés, comme ils le seraient si la région n'était pas amplifiée;

- enfin les gènes dont l'expression est préjudiciable à la prolifération de cette sous-population cellulaire, et qui sont amplifiés car trop proches du gène important, faisant en quelque sorte obligatoirement partie des amplicons. L'amplification de ces gènes devrait logiquement s'accompagner d'une expression suffisamment faible pour ne pas s'opposer à l'avantage sélectif lié à l'amplification du gène important.

Dans le premier groupe, on peut placer $N$-myc, dont la position centrale dans les amplicons conduit, en plus des arguments statistiques et cliniques, à en faire le gène important de la région concernée [25]. De même, l'amplification de la région 17q11-q12 est-elle probablement à mettre au crédit de $c$-erbB-2, qui, outre les propriétés de sa protéine, voit son amplification accompagnée d'une hyperexpression, en tout cas dans les cancers du sein et de l'ovaire [26]. c-erbA-1, également situé dans la même région chromosomique mais moins fréquemment amplifié dans les cancers du sein, et seulement lorsque $c$-erbB-2 l'est, voit son amplification ne s'accompagner d'aucune expression détectable [27]. Cela le place plutôt dans la catégorie des gènes indifférents ou néfastes.

D'autres exemples de gènes présents sur un même amplicon ont été décrits. Ainsi une partie du gène $P V T$ est amplifiée avec c-myc dans plusieurs lignées cellulaires provenant de carcinomes coliques [28]. Il existe également une unité transcriptionnelle active coamplifiée tant avec le gène DHFR [29], qu'avec celui de l'adénylate déaminase [30]. Ces co-amplifications sont-elles fortuites, ou ont-elles une signification fonctionnelle?

Les gènes int-2 et hst, situés en $11 \mathrm{q} 13$ à environ 40 kilobases l'un de l'autre, sont co-amplifiés dans $17 \%$ des cancers primitifs du sein [31], ainsi que dans d'autres cancers (voies aérodigestives supérieures, œsophage, mélanome malin). Il est tentant de faire de l'un de ces deux gènes, ou des deux, les responsables de la sélection de l'amplification constatée, 
compte tenu de leur appartenance à la famille des facteurs de croissance fibroblastiques, de leurs propriétés oncogéniques et du rôle de leurs homologues murins dans la cancérogenèse mammaire viro-induite de la souris [32]. Pourtant, l'expression de ces gènes est difficilement détectable, et il est très possible qu'elle ne soit pas liée à leur amplification [33, 34]. De plus, l'existence de plusieurs tumeurs du sein dans lesquelles le locus bcl-1, point de cassure impliqué dans les translocations $t(11 ; 14)$ caractéristiques de certaines hémopathies malignes, et situé également en $11 \mathrm{q} 13$, est amplifié sans que int-2 et hst le soient, ou à un niveau plus élevé que ceux-ci [31], pose la question de l'existence d'un autre gène participant à la sélection des amplicons trouvés en 11q13, ou d'une partie de ceux-ci.

Il n'est donc pas toujours évident de définir quel est le gène important dans un amplicon. Si les proto-oncogènes sont de bons candidats, il est aussi possible que d'autres gènes, situés à proximité d'un proto-oncogène, puissent participer à l'évolution tumorale, par exemple en étant impliqués dans des voies métaboliques conduisant à, ou activées par, l'expression d'un proto-oncogène, et pourquoi pas de celui situé sur le même amplicon : une sorte d'" amplicancéron " serait ainsi sélectionnée au cours de l'évolution tumorale. Il a été récemment constaté que certaines HSR détectées dans des cancers primitifs du sein ne contiennent aucun des proto-oncogènes habituellement retrouvés dans ce type de maladie [35]. On peut imaginer que certaines régions amplifiées au cours de l'évolution tumorale ne contiennent pas de proto-oncogène au sens strict, mais plutôt un ou plusieurs gènes dont l'hyperexpression pourrait permettre à un cancer d'évoluer vers une forme plus invasive

\section{TIRÉS A PART}

P. Gaudray.

\section{Summary}

Amplification, proto-oncogene and human cancers

Amplification is one possible way for proto-oncogene activation in some human cancers. Intra- or extra-chromosomal amplification of a defined DNA fragment seems to involve recombination events which could be related - or identical to those leading to other genetic alterations, such as deletions or translocations. The amplicon, which seems to be large in most instances, contains several other genes neighboring the selected one. A possible role of these co-amplified genes in tumor progression remains to be established. In human neuroblastomas, $N$-myc amplification seems to be a good marker of bad prognosis. Pronostic value is more controversial in most cases of protooncogene amplification/human tumor couples. 